\title{
ESTEROIDES SUBTENONIANOS EN NEOVASCULARIZACIÓN COROIDEA SECUNDARIA A COROIDITIS SERPINGINOSA. PRESENTACIÓN DE UN CASO
}

\author{
SUB-TENON STEROID TREATMENT IN CHOROIDAL \\ NEOVASCULARIZATION CAUSED BY SERPIGINOUS \\ CHOROIDITIS. A CASE REPORT
}

\author{
PARROCHIA M ${ }^{1}$, MARTÍN-AVIÀ J ${ }^{2}$, MARTÍNEZ-CASTELLANOS MA ${ }^{1}$, NAVARRO P ${ }^{1}$, \\ ARELLANES L ${ }^{1}$, QUIROZ-MERCADO $\mathrm{H}^{1}$
}

\begin{abstract}
RESUMEN
Caso clínico: Se presenta el caso clínico de una paciente con coroiditis serpiginosa y neovascularización coroidea (NVC) activa en ambos ojos. Tras tratamiento con terapia inmunosupresiva sistémica junto con inyección sub-Tenon posterior de esteroides, conseguimos la resolución de la NVC en ambos ojos, mejorando la capacidad visual en uno de los ojos.

Discusión: La NVC es una complicación de las uveítis posteriores, cuya presencia amenaza de forma importante la visión. El presente caso sugiere que los esteroides subtenonianos añadidos a terapia inmunosupresiva sistémica, pueden acelerar la resolución de NVC tras uveítis posteriores.
\end{abstract}

Palabras clave: Coroiditis serpiginosa, neovascularización retiniana, esteroides, subtenon, inmunosupresora.

\begin{abstract}
Case report: We present a report of a patient suffering from serpiginous choroiditis complicated by choroidal neovascularization (CNV). Sub-tenon steroid treatment, added to the usual immunosuppressive therapy, resolved the $\mathrm{CNV}$, improving visual acuity in one eye.

Discussion: Choroidal neovascularization is a sight-threatening complication of posterior uveitis. Our case suggests that sub-tenon steroid treatment, added to systemic immunosuppressive therapy, may accelerate the $\mathrm{CNV}$ resolution in posterior uveitis (Arch Soc Esp Oftalmol 2006; 81: 615-620).
\end{abstract}

Key words: Serpiginous choroiditis, retinal neovascularization, steroids, sub-tenon, immunosuppressive.

Recibido: 7/11/05. Aceptado: 21/9/06.

Hospital Luis Sánchez Bulnes. Asociación para Evitar la Ceguera en México, México DF, México.

1 Licenciado en Medicina.

2 Licenciado en Medicina. Hospital Clínico Universitario Lozano Blesa. Zaragoza. España.

Correspondencia:

María Ana Martínez Castellanos

Servicio de Retina. Hospital Luis Sánchez Bulnes, APEC.

Vicente García Torres, 46

México DF 04330

México

E-mail: mamc@dr.com 


\section{INTRODUCCIÓN}

La coroiditis serpiginosa es un desorden progresivo y poco frecuente que envuelve la coriocapilar, el epitelio pigmentario de la retina y la retina neurosensorial. La enfermedad se inicia de forma aguda en gente joven y de mediana edad, principalmente. La clínica varía desde una pérdida indolora de la visión hasta un escotoma central (1). Habitualmente se presenta con visión borrosa moderada o metamorfopsia, ya que la fóvea no suele afectarse de inicio (1).

El curso de la enfermedad se caracteriza por episodios recurrentes que pueden ser asintomáticos mientras no exista afectación foveal (1). Las lesiones subretinianas amarillentas características se inician típicamente en la zona peripapilar, esparciéndose de forma centrífuga, afectando finalmente a la fóvea. Con el tiempo, la enfermedad casi siempre afecta a ambos ojos, de forma asimétrica.

La angiografía fluoresceínica (AGF) demuestra un patrón característico de bloqueo temprano con hiperfluorescencia tardía en los bordes de las lesiones activas (1). La neovascularización coroidea (NVC) es una complicación descrita con una incidencia de un 10 a un $25 \%$ de los casos (1).

La opinión generalizada es que la terapia esteroidea sistémica o periocular suele ser efectiva en frenar la inflamación activa y en acortar la duración del episodio (1-4). Los fármacos inmunosupresores descritos para el tratamiento de la coroiditis serpiginosa incluyen la ciclosporina, combinaciones de prednisona-ciclosporina, azatioprina y terapia con agentes alquilantes junto con ciclofosfamida o clorambucil.

Los corticoesteroides tienen propiedades antiangiogénicas, antifibrinolíticas y antipermeabilizantes.
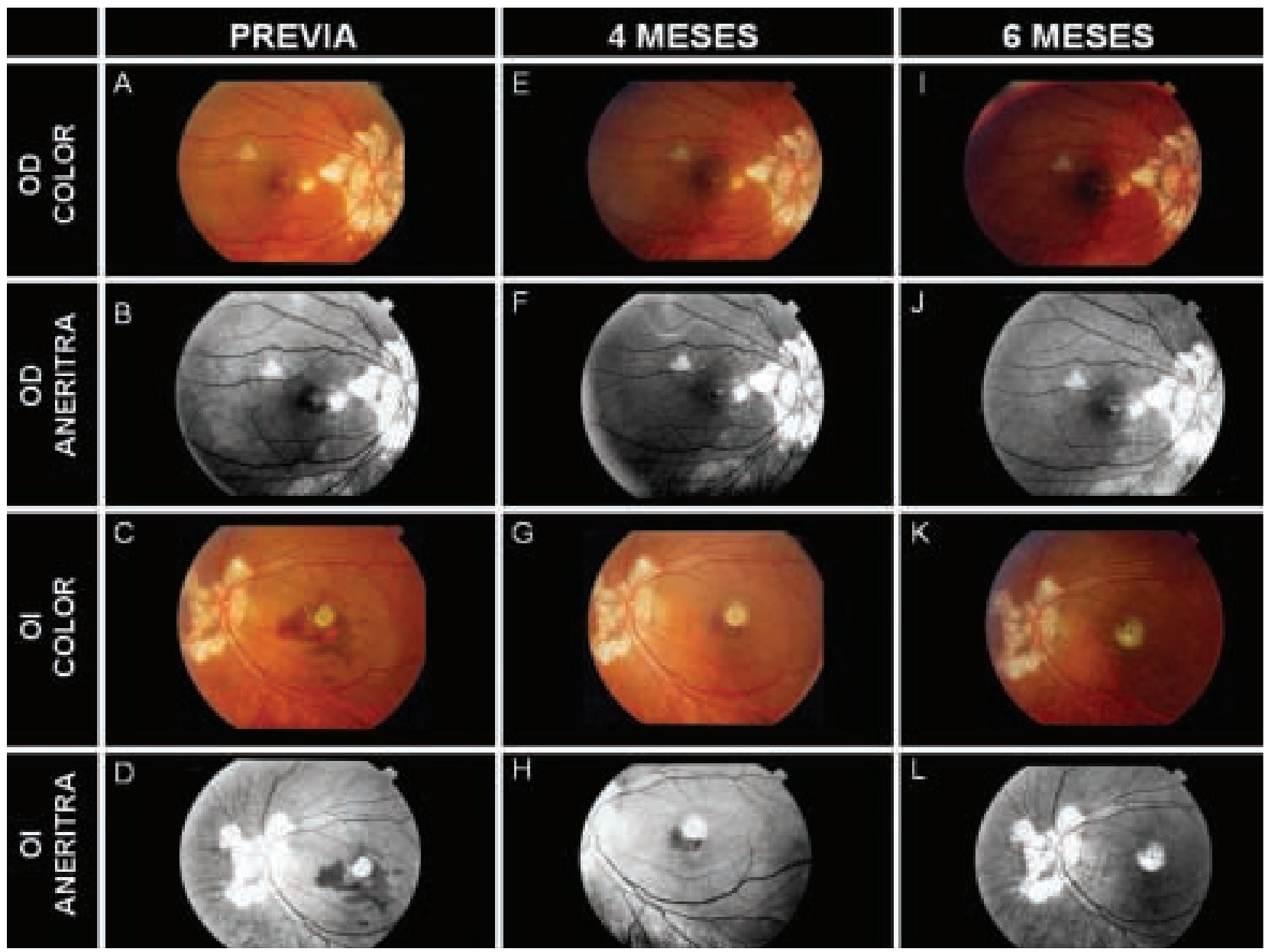

Fig. 1: Composición fotográfica de fotografías de fondo a color y aneritra de ambos ojos comparativas, previo al tratamiento, a los 4 meses y a los seis meses. 
Aunque sus principales efectos son la estabilización de la barrera hemato-retiniana, la reabsorción de exudados y la regulación del estímulo inflamatorio, también son potentes inhibidores de la neovascularización (4). Se han propuesto muchos mecanismos de inhibición de la angiogénesis por los esteroides, incluyendo una alteración de la composición de la membrana basal capilar, suprimiendo su desaparición y la inhibición de la migración celular endotelial (5).

Los corticoides actúan en la cascada neovascular mediante la disminución directa de los niveles del factor de crecimiento vascular endotelial (VEGF), inhibiendo factores de crecimiento fibroblásticos y actuando sobre los macrófagos que los liberan (5).

Nuestro propósito es describir el caso de una NVC secundaria a coroiditis serpiginosa, tratada con éxito mediante esteroides en inyección subTenon posterior junto con azatioprina.

\section{CASO CLÍNICO}

Se trata de paciente de 61 años de edad, de raza hispana, originaria de Veracruz, México, sin antecedentes patológicos de interés. Acude a nuestro centro por disminución de visión en ambos ojos (AO) de 3 años de duración. Su capacidad visual (CV) en OD era de 0,2 y de cuenta dedos a 4 metros en OI. En la exploración fundoscópica encontramos en $\mathrm{AO}$ alteraciones coroideas peripapilares, compatibles con una coroiditis serpiginosa inactiva. En el OD se apreciaba una lesión coroidea activa temporal a la mácula con NVC subfoveal. En el OI, se observaba una cicatriz disciforme subfoveal inactiva, aunque aparentaba un borde activo inferior, extrafoveal. (figs. 1A, 1C). Se confirmaron los hallazgos mediante AGF (figs. $2 \mathrm{~A}, 2 \mathrm{~B}, 2 \mathrm{C}$ y $3 \mathrm{~A}, 3 \mathrm{~B}, 3 \mathrm{C})$.
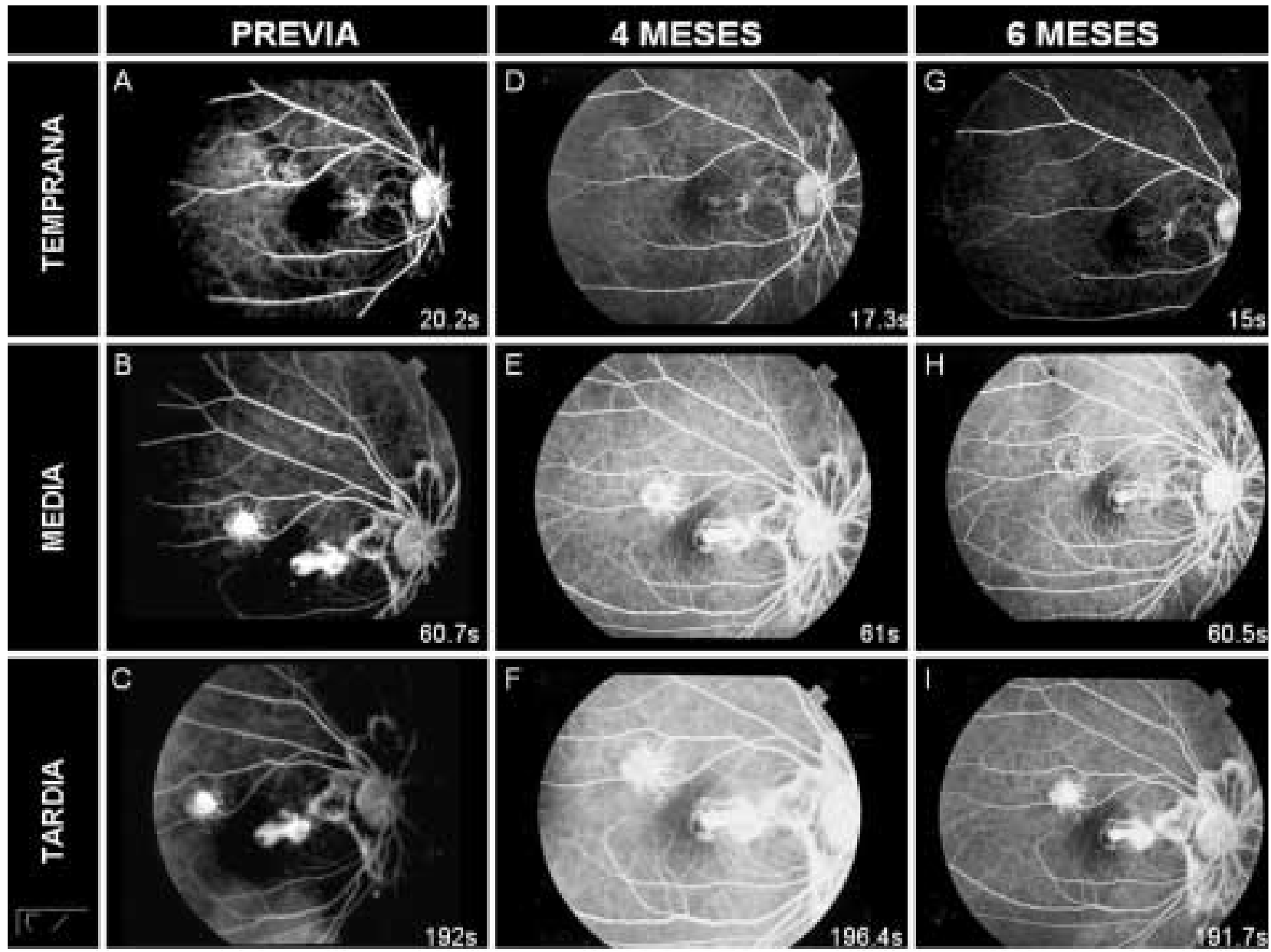

Fig. 2: Composición fotográfica de angiografía fluoresceínica de ojo derecho en fases iniciales, medias y tardías comparativas previo al tratamiento a los 4 meses y a los 6 meses. 
Se inició tratamiento mediante azatioprina y prednisona $50 \mathrm{mg} / \mathrm{d}$ ía. No se aplicó terapia fotodinámica por limitaciones económicas del paciente y por no contar con seguridad social. Un mes más tarde se decidió añadir corticoides subtenonianos (fosfato y acetato de betametasona). Dos meses tras el inicio del tratamiento, se inyectó una segunda dosis subtenoniana de corticoides. En el control realizado 4 meses después de la segunda inyección, la NVC se resolvió en el OD con reabsorción del líquido submacular, aunque en el OI persistía el borde inferior activo de la cicatriz subfoveal (fig. 1E y 1G) confirmado por AGF (fig. 2D, 2E, 2F y 3D, 3E, 3F). Tras 6 meses de tratamiento, se resolvió la NVC en OI, habiendo mejorado la CV del OD a 3/10, sin variar en el OI (fig. 1I, $1 \mathrm{~K}, 2 \mathrm{G}, 2 \mathrm{H}, 2 \mathrm{I}$ y $3 \mathrm{G}, 3 \mathrm{H}$, 3I).

\section{DISCUSIÓN}

La proliferación de tejido vascular intraocular es uno de los problemas más importantes en la actualidad en la clínica oftalmológica. Los corticoesteroides han demostrado claramente la reducción de la inflamación y la supresión de la proliferación celular. Así pues, los esteroides se han usado para el tratamiento de muchas enfermedades oculares, administrados de forma local o sistémica. Teniendo en cuenta la presencia de los efectos secundarios sistémicos y que el ojo sólo ocupa el $0,01 \%$ del volumen corporal, es más efectivo la aplicación de los esteroides directamente en la región en la que se requiera su acción.

En humanos, se ha descrito el uso de esteroides sistémicos, subtenonianos e intravítreos para el trata-
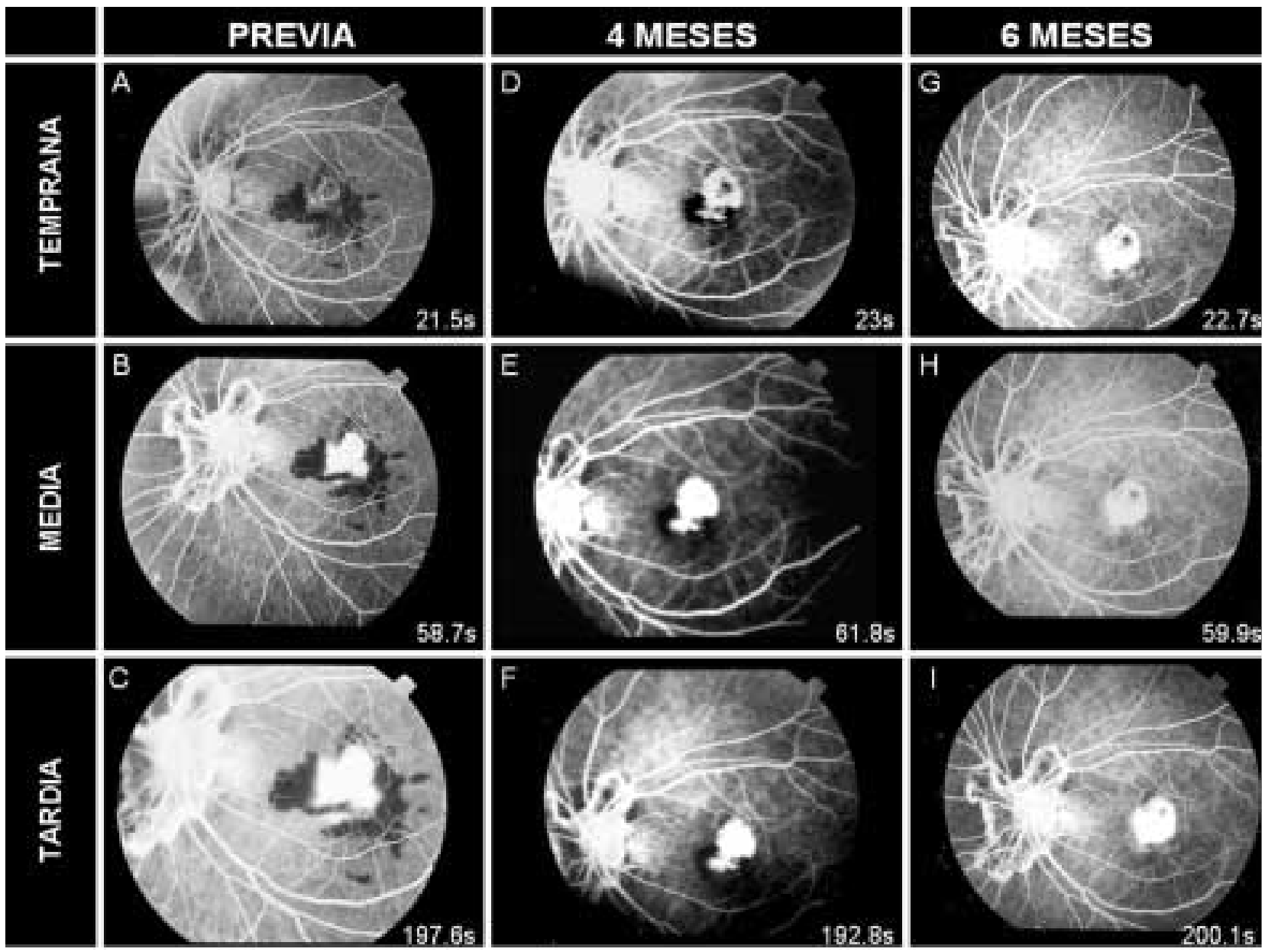

Fig. 3: Composición fotográfica de angiografía fluoresceínica de ojo izquierdo en fases iniciales, medias y tardías comparativas previo al tratamiento a los 4 meses y a los 6 meses. 
miento de la NVC en el síndrome de histoplamosis ocular, aunque todavía no se han realizado estudios controlados. Igualmente se ha descrito que el tratamiento con ciclosporina A y esteroides pueden ser beneficiosos en la resolución de la NVC en la coroiditis serpiginosa, con resultados a los 6 meses (4).

Las publicaciones de tratamiento con esteroides en coroiditis serpinginosa, usualmente tratan la coroiditis serpinginosa activa (5). Nuestro paciente presenta una membrana neovascular coroidea activa bilateral secundaria a la coroiditis serpinginosa, pero sin actividad inflamatoria, por lo que se decide a no aplicar terapia intravítrea con acetónido de triamcinolona ya que preferimos su uso en la coroiditis activa (5). En últimas fecha se encuentra un auge en el uso de antiangiogénicos, con excelentes resultados en neovascularización coroidea, pero la experiencia en neovascularización secundaria a un proceso inflamatorio es limitada, por lo que no consideramos su uso en un paciente con trastorno bilateral.

El resultado del presente caso clínico sugiere que el uso de acetato y fosfato de betametasona admi- nistrado vía subtenoniana añadido al tratamiento inmunosupresor sistémico, puede ser útil para estabilizar y mejorar la capacidad visual en pacientes con NVC tras coroiditis serpinginosa, acelerando su resolución.

\section{BIBLIOGRAFÍA}

1. Akpek EK, Ilhan-Sarac O. New treatments for serpiginous choroiditis. Curr Opin Ophthalmol 2003; 14: 128-131.

2. Ciulla TA, Walker JD, Fong DS, Criswell MH. Corticosteroids in posterior segment disease: an update on new delivery systems and new indications. Curr Opin Ophthalmol 2004; 15: 211-220.

3. Pathengay A. Intravitreal triamcinolone acetonide in serpinginous choroidopathy. Indian J Ophthalmol 2005; 53: 77-79.

4. Vianna RN, Ozdal PC, Deschenes J, Burnier MN Jr. Combination of azathioprine and corticosteroids in the treatment of serpinginous choroiditis. Can J Ophthalmol 2006; 41: 183-189.

5. Lim WK, Buggage RR, Nussenblatt RB. Serpinginous choroiditis. Surv Ophthalmol 2005; 50: 231-244. 